\title{
Phylogenetic analysis and rapid identification of Candida dubliniensis based on analysis of $A C T 1$ intron and exon sequences
}

\author{
Samantha M. Donnelly, Derek J. Sullivan, Diarmuid B. Shanley \\ and David C. Coleman \\ Author for correspondence: Derek J. Sullivan. Tel: +35316126275 . Fax: +35316711255 . \\ e-mail : djsullvn@dental.tcd.ie
}

Department of Oral Medicine and Oral Pathology, School of Dental Science, University of Dublin, Trinity College, Dublin 2, Republic of Ireland
The phylogenetic position of Candida dubliniensis has previously been established on the basis of the sequence of rRNA genes. In order to confirm the relationship between $C$. dubliniensis and other yeast species, particularly Candida albicans, using non-rRNA gene sequences the ACT1 gene was chosen for analysis. Three overlapping fragments that together span the entire $C$. dubliniensis ACT1 gene (CdACT1) were amplified from a recombinant phage isolated from a genomic DNA $\lambda$ library using PCR. These were cloned and used to determine the contiguous sequence of the gene. Analysis of the sequence data revealed the presence of a 1131 bp ORF interrupted by a single 632 bp intron at the 5' extremity of the gene. Comparison of the CdACT1 sequence with the $\mathrm{C}$. albicans homologue (CaACT1) revealed that although the exons are $97.9 \%$ identical the introns are only $83.4 \%$ identical. Phylogenetic trees generated using $A C T 1$ exon and intron sequences from a range of yeast species unequivocally confirmed the phylogenetic position of $C$. dubliniensis as a unique taxon within the genus Candida. Analysis of the ACT1-associated intron sequences from 10 epidemiologically unrelated $C$. dubliniensis isolates from disparate geographical locations showed a very low level of intraspecies sequence variation. In order to develop an accurate and rapid method to identify $C$. dubliniensis from primary isolation plates the significant divergence between the $C$. dubliniensis and $C$. albicans $A C T 1$ intron sequences was exploited by designing C. dubliniensis-specific PCR primers. Using a rapid boiling method to produce template DNA directly from colonies from primary isolation plates in $10 \mathrm{~min}$, these primers were used in a blind test with 122 isolates of C. dubliniensis, 53 isolates of C. albicans, 10 isolates of C. stellatoidea and representative isolates of other clinically relevant Candida and other yeast species. Only the $C$. dubliniensis isolates yielded the $C$. dubliniensis-specific $\mathbf{2 8 8}$ bp amplimer. Use of this technique on colonies suspected to be $C$. dubliniensis allows their correct identification as $C$. dubliniensis in as little as $4 \mathrm{~h}$.

Keywords: ACT1, phylogenetics, Candida dubliniensis, PCR identification

\section{INTRODUCTION}

Candida dubliniensis is a yeast species first described in 1995 (Sullivan et al., 1995). Although C. dubliniensis is

\section{Abbreviations: HIV, human immunodeficiency virus.}

The EMBL accession numbers for the nucleotide sequences reported in this paper are AJ236897 (Candida dubliniensis CD36), AJ237918 (Candida tropicalis NCPF 3111) and AJ237919 (Candida stellatoidea ATCC 11006). phenotypically similar to C. albicans, the two species differ significantly at the genetic level. In particular, phylogenetic analysis of large- and small-subunit rRNA gene sequences provided the basis for the designation of C. dubliniensis as a separate species (Sullivan et al., 1995, 1997; Gilfillan et al., 1998). The first isolates identified as C. dubliniensis were recovered from the oral cavities of Irish human immunodeficiency virus (HIV)-infected individuals. However, over the last three 
years there have been increasing numbers of reports of the recovery of $C$. dubliniensis isolates by laboratories throughout the world, including Europe, North and South America, and Australia (Coleman et al., 1997b; Sullivan et al., 1997; Sullivan \& Coleman, 1998; Salkin et al., 1998; Kirkpatrick et al., 1998). Although the majority of these isolates have been primarily associated with oral candidosis in HIV-infected and AIDS patients (Coleman et al., 1997a), C. dubliniensis isolates have also been recovered form the oral cavities, gastrointestinal tracts and vaginas of HIV-negative individuals (Moran et al., 1997, 1998; Sullivan \& Coleman, 1998; Odds et al., 1998). There have also been reports of $C$. dubliniensis isolates associated with systemic disease (Pinjon et al., 1998; Meis et al., 1999). The majority of clinical isolates of C. dubliniensis have been shown to be susceptible to commonly used antifungal drugs, including fluconazole (Moran et al., 1997, 1998; Kirkpatrick et al., 1998; Pfaller et al., 1999). However, fluconazole resistance has been detected in clinical isolates (Moran et al., 1997, 1998; Kirkpatrick et al., 1998; Pfaller et al., 1999) and isolates of C. dubliniensis susceptible to fluconazole can be readily induced to produce fluconazole-resistant derivatives following exposure to the drug in vitro (Moran et al., 1997, 1998).

The prevalence of $C$. dubliniensis in the oral cavities of HIV-infected individuals and AIDS patients and reports of its association with disease in other body sites warrant in-depth epidemiological analysis. However, these investigations have been hampered by the lack of a simple, reliable method capable of unequivocally differentiating between C. dubliniensis and C. albicans in the clinical laboratory. Indeed, since C. dubliniensis and C. albicans share the ability to produce germ tubes and chlamydospores, features previously used for the definitive identification of C. albicans, it is likely that many isolates of C. dubliniensis have been misidentified as C. albicans. Investigations of our own collection of stored oral Candida isolates, originally identified as C. albicans, have shown that $1.8 \%$ of isolates recovered from asymptomatic normal healthy individuals and $16.5 \%$ of isolates recovered from HIV-infected individuals were in fact C. dubliniensis (Coleman et al., 1997a). In a similar study, Odds et al. (1998) have recently shown that approximately $2 \%$ of a stored archival culture collection of 2500 yeast isolates, originally identified as C. albicans, was C. dubliniensis. They found that the prevalence of C. dubliniensis was significantly higher among HIV-infected individuals than among HIVnegative individuals (Odds et al., 1998). Although first described in 1995, the earliest known C. dubliniensis isolates were recovered in the $1950 \mathrm{~s}$, thus predating the HIV pandemic. One of these strains, NCPF 3108, was recovered in the UK in 1957 and was originally deposited in the British National Collection for Pathogenic Fungi as C. stellatoidea (Sullivan et al., 1995), while another strain, CBS 2747, which was recovered in the Netherlands in 1952, was originally deposited in the Centraal Bureau fur Schimmelcultures as C. albicans (Meis et al., 1999).
A variety of tests have been developed to discriminate between C. dubliniensis and C. albicans based upon phenotypic characteristics. These include carbohydrate assimilation profiles and colonial coloration on differential media such as CHROMagar Candida and methyl blue-Sabouraud agar (Sullivan et al., 1995, 1997; Coleman et al., 1997a; Schoofs et al., 1997). However, some of these assays have been shown to be unreliable in some instances and should only be used for the presumptive identification of C. dubliniensis from clinical specimens (Schoofs et al., 1997; Sullivan \& Coleman, 1998; Kirkpatrick et al., 1998). The accuracy of C. dubliniensis isolate identification based on carbohydrate assimilation profiles has been improved by the recent inclusion of the assimilation profiles of some C. $d u b$ liniensis strains in the databases of commercially available yeast identification systems, including the API ID $32 \mathrm{C}$ and 20C AUX systems. It has been reported recently that C. dubliniensis and C. albicans can be distinguished on the basis of differential growth at $45^{\circ} \mathrm{C}$, with isolates of the former species unable to grow at this temperature (Pinjon et al., 1998). However, in a recent study a significant number of C. albicans isolates were found to be unable to grow at this temperature (Kirkpatrick et al., 1998). Currently, the most reliable tests available to differentiate between these species are based on molecular techniques such as DNA fingerprinting with repetitive-sequence-containing probes, randomly amplified polymorphic DNA (RAPD) analysis and pulsedfield gel electrophoresis (Sullivan et al., 1995), but these are not suitable for the analysis of large sample numbers in routine diagnostic laboratories. However, since the differences between C. dubliniensis and C. albicans are most pronounced at the genetic level such differences should provide the basis for a specific and rapid identification test. One molecular technique with the required degree of specificity and ease of use is the polymerase chain reaction (PCR). This technology is increasingly available in diagnostic laboratories and due to its speed, reproducibility and high sample volume throughput is ideally suited for application to large numbers of clinical isolates.

The phylogenetic position of C. dubliniensis in relation to other yeast species has been established on the basis of the comparison of small- and large-subunit rRNA gene sequences (Sullivan et al., 1995, 1997; Gilfillan et al., 1998). In the present study we sought to confirm these phylogenetic relationships using sequences of nonrRNA gene origin. It was also hoped that these sequence data would lead to the identification of C. dubliniensisspecific nucleotide sequences that could be exploited in the design of a rapid PCR-based identification test. To achieve these goals the ACT1 gene of C. dubliniensis was chosen for analysis. ACT1 encodes actin, a protein that is abundant in all eukaryotic cells, where it is the major component of cytoplasmic microfilaments. Due to structural constraints the amino acid sequence of actin proteins from different eukaryotic species is highly conserved (Korn et al., 1978; Hightower et al., 1986; Pollard et al., 1990; Hennessey et al., 1993; Welch et al., 
1994). Since C. albicans and C. dubliniensis are very closely related it was anticipated that the ACT1 genes of these species would be very similar. Results presented in this study for C. dubliniensis and in a previous study for C. albicans (Losberger \& Ernst, 1989) showed that both ACT1 genes contain a single class IV intron and it was anticipated that these intron sequences would be subject to less evolutionary conservation than the actin-proteincoding exons. Therefore we decided to investigate whether the exons and introns of C. albicans and $C$. dubliniensis would be sufficiently divergent to allow an accurate determination of the phylogenetic relationship between the two species and to allow the design of $C$. dubliniensis-specific primers suitable for rapid and specific identification of this species in the clinical laboratory using a rapid template DNA preparation procedure.

\section{METHODS}

Candida strains and culture media. All C. dubliniensis strains were isolated by this laboratory or received from other laboratories and identified using the molecular and phenotypic methods described by Sullivan et al. (1995). All Candida strains and isolates were routinely grown on Potato Dextrose Agar (PDA, Oxoid) at $\mathrm{pH} 5.6$ for $48 \mathrm{~h}$ at $37^{\circ} \mathrm{C}$. For liquid culture, Candida strains and isolates were grown at $37^{\circ} \mathrm{C}$ in Yeast Peptone Dextrose Broth (YPD) in an orbital incubator (Gallenkamp) set at 150 r.p.m.

Bacterial strains and culture media. Escherichia coli DH5 $\alpha$ was used as the host strain for phagemid pBluescript II $\mathrm{KS}(+/-)$ (Stratagene) and was maintained on Luria-Bertani (LB) agar, supplemented with $100 \mu \mathrm{g}$ ampicillin $\mathrm{ml}^{-1}$ to maintain plasmids where appropriate. For liquid culture, strains harbouring plasmids were grown at $37^{\circ} \mathrm{C}$ in LB broth containing $100 \mu \mathrm{g}$ ampicillin $\mathrm{ml}^{-1}$ in an orbital incubator set at
150 r.p.m. Transformation of E. coli DH5 $\alpha$ and identification of transformants containing recombinant plasmids were carried out by standard protocols (Sambrook et al., 1989). E. coli LE 392 and its P2 phage lysogenic derivative (P2 392) were used for propagating the bacteriophage $\lambda$ cloning vector EMBL3 and its recombinant derivatives. These strains were grown and maintained on LB agar supplemented with $10 \mathrm{mM}$ $\mathrm{MgSO}_{4}$ and $0.2 \%(\mathrm{w} / \mathrm{v})$ maltose. Organisms for phage infection were grown in $\mathrm{LB}$ broth containing $10 \mathrm{mM} \mathrm{MgSO}_{4}$ and $0 \cdot 2 \%(\mathrm{w} / \mathrm{v})$ maltose (Sambrook et al., 1989).

Chemicals, enzymes, radioisotopes and oligonucleotides. Analytical-grade or molecular-biology-grade chemicals were purchased from Sigma-Aldrich, BDH or Boehringer Mannheim. Enzymes were purchased from Promega or Boehringer Mannheim and used according to the manufacturer's instructions. $\left[\alpha-{ }^{32} \mathrm{P}\right] \mathrm{dATP}\left(3000 \mathrm{Ci} \mathrm{mmol}{ }^{-1} ; 110 \mathrm{TBq} \mathrm{mmol}^{-1}\right)$ was purchased from Amersham. Custom-synthesized oligonucleotides were purchased from Genosys Biotechnologies (Europe).

DNA extraction procedures. Plasmid DNA for restriction endonuclease digestion and Southern hybridization was prepared by the alkaline lysis method described by Sambrook et al. (1989). Plasmid DNA for sequencing was prepared using a Quantum Prep Plasmid Miniprep kit (Bio-Rad). Total cellular DNA from Candida isolates was prepared as described by Gallagher et al. (1992). High-molecular-mass total cellular DNA from C. dubliniensis for the construction of a genomic library was isolated by the method described by Bennett $e t$ al. (1998). Candida template DNA for use in PCR experiments with the C. dubliniensis-specific oligonucleotide primer pair DUBF/DUBR (Table 1) was prepared as follows. A single colony from a culture grown for $48 \mathrm{~h}$ at $37^{\circ} \mathrm{C}$ on PDA or CHROMagar Candida medium (CHROMagar Candida, Paris, France) was suspended in $50 \mu \mathrm{l}$ sterile distilled water. Cell suspensions were boiled for $10 \mathrm{~min}$ and the lysed cells subjected to a clearing spin for $5 \mathrm{~min}$ at $20000 \mathrm{~g}$. Template DNA contained in $25 \mu \mathrm{l}$ supernatant was used for PCR amplification.

Table 1. PCR primers used in this study

\begin{tabular}{|c|c|c|c|}
\hline Primer & Sequence & $\begin{array}{c}\text { Nucleotide co- } \\
\text { ordinates }\end{array}$ & RE site $†$ \\
\hline \multicolumn{4}{|c|}{ C. albicans } \\
\hline $5^{\prime} \mathrm{F}$ & 5'-CGGAATTCCTTAGAAACATTATCTCGAT-3' & -49 to -30 & EcoRI \\
\hline $5^{\prime} \mathrm{R}$ & 5'-GCICTAGAGAGAAATATTATGTCGACAA-3' & 126 to 145 & $X b a \mathrm{I}$ \\
\hline ACTF & 5'-CGGAATTCAATGGACGGTGGTATGTT-3' & -1 to 17 & EcoRI \\
\hline ACTR & 5'-CGGAATTCAATGGATGGACCAGATTCGTCG-3' & 1746 to 1767 & EcoRI \\
\hline $3^{\prime} \mathrm{F}$ & 5'-CGGAATTCTAAGATTATTGCTCCACCAG-3' & 1641 to 1660 & EcoRI \\
\hline $3^{\prime} \mathrm{R}$ & 5'-GCTCTAGACCAGATTTCCAGAATTTCAC-3' & 1792 to 1811 & $X b a \mathrm{I}$ \\
\hline INTF & 5'-CGGAATTCAATGGACGGTGGTATGGT-3' & -1 to 17 & EcoRI \\
\hline INTR & 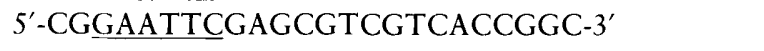 & 724 to 739 & EcoRI \\
\hline \multicolumn{4}{|c|}{ C. dubliniensis } \\
\hline DUBF & $5^{\prime}$-GTATTTGTCGTTCCCCTTTC-3' & 251 to 270 & \\
\hline DUBR & $5^{\prime}$-GTGTTGTGTGCACTAACGTC-3' & 519 to 538 & \\
\hline
\end{tabular}

"Primers were complementary to ACT1 gene sequences as follows: CaACT1, accession no. X16377 (Losberger \& Ernst, 1989); and CdACT1, accession no. AJ236897 (this study). Nucleotide co-ordinates shown are numbered in the $5^{\prime}$ to $3^{\prime}$ direction with the first base of the translation start codon being +1 .

† Restriction endonuclease recognition sequence included within the primer sequence (underlined). 


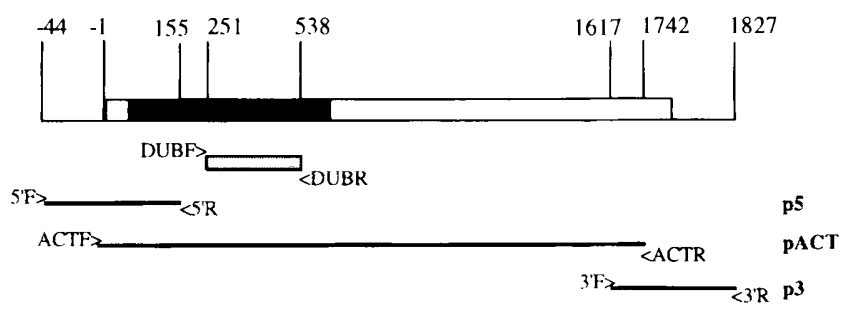

Fig. 1. Schematic diagram of the $C$. dubliniensis ACT1 gene (CdACT1), which is represented by the large horizontal box. The black area corresponds to the position of the intron at the 5 end of the gene. The thin horizontal lines in the lower part of the figure represent sequences amplified from the CdACT1encoding recombinant phage $\triangle C D A C T 1$ using the primer pairs $5^{\prime} F / 5^{\prime} R, A C T F / A C T R$ and $3^{\prime} F / 3^{\prime} R$ (Table 1). The names of the recombinant plasmids obtained when these amplimers were cloned in pBluescript are shown on the right. The location of sequences amplified with the $C$. dubliniensis-specific primer pair DUBF/DUBR is indicated by the shaded box. The nucleotide coordinates of the sequences contained in each amplimer relative to CdACT1 sequences are shown at the top (numbering the CdACT1 ATG start codon +1).

Large-scale E. coli phage lysates were prepared according to the plate method of Sambrook et al. (1989) and recombinant phage DNA was purified from phage preparations using a Wizard Lambda Preps kit (Promega).

Construction of a C. dubliniensis CD36 genomic DNA library. The DNA library was constructed by ligating Sau3Agenerated partial digestion products of C. dubliniensis CD36 chromosomal DNA $>10 \mathrm{~kb}$ in size with BamHI-generated pre-prepared; bacteriophage replacement vector EMBL3 arms (Promega) followed by packaging in vitro into preprepared phage heads and tails (Promega) according to the manufacturer's instructions. Previous studies have shown that DNA fragments ranging in size from 9 to $23 \mathrm{~kb}$ can be cloned into the EMBL3 vector (Frischauf et al., 1983). The packaged recombinant phage particles were propagated on the E. coli lysogenic strain P2 392. A recombinant library containing $2 \cdot 0$ $\times 10^{5}$ p.f.u. was obtained.

Recombinant phages were propagated on E. coli LE 392 to yield 1000 p.f.u. per $90 \mathrm{~mm}$ Petri plate and were transferred from the plaques onto nitrocellulose membrane filters (Schleicher \& Schuell) by overlaying the plaques with the filters, which were then screened by plaque hybridization (Sambrook et al., 1989) using $\alpha$ - ${ }^{32}$ P-labelled DNA probes.

DNA hybridization. Probe DNA used in screening the $C$. dubliniensis genomic DNA library and in Southern hybridization experiments was labelled with $\left[x-{ }^{32} \mathrm{P}\right] \mathrm{dATP}(3000 \mathrm{Ci}$ $\mathrm{mmol}^{-1} ; 110 \mathrm{TBq} \mathrm{mmol}^{-1}$ ) by random priming with a random hexanucleotide primer labelling kit (Prime-a-Gene, Promega). All hybridizations were performed under conditions of high stringency $\left(65^{\circ} \mathrm{C}\right)$, as described by Sambrook et al. (1989).

PCR isolation of ACT1-associated introns. Amplification of ACT1-associated intron sequences from Candida strains was performed in $100 \mu \mathrm{l}$ reaction mixtures containing $100 \mathrm{pmol}$ each of the forward and reverse primers, INTF/INTR (Table 1), $250 \mu \mathrm{M}$ deoxynucleotide triphosphates, $2.0 \mathrm{mM} \mathrm{MgSO}$, $20 \mathrm{mM}$ Tris $/ \mathrm{HCl}\left(\mathrm{pH} 8.8\right.$ at $\left.25^{\circ} \mathrm{C}\right), 10 \mathrm{mM} \mathrm{KCl}, 10 \mathrm{mM}$ $\left(\mathrm{NH}_{4}\right)_{2} \mathrm{SO}_{4}, 0.1 \%(\mathrm{v} / \mathrm{v})$ Triton X-100, $1 \mathrm{U}$ Vent $_{\mathrm{k}}$ DNA polymerase (New England Biolabs) and $500 \mathrm{ng}$ template DNA. PCR reactions were performed in a DNA Thermal Cycler
作 CGATAGAGTGTTGTTGTTTAGATCTGGGTGGGAAAGAACCCATTTCCATTCAGATCAAG

Ca CTTTTTTGTTGTCGACATAATATTTCTCGTTMGGATGTTACTGTCACATTAATAATACA -TTTTTTGTTGTCGACATAATATTTCTCGTTTGGGATGTTACTGTCACATTA-----ACA

CACACATCAGCTTATAATTTTGAAAGTAATTTATCAGATATGTTGTGACGATCAATGGAA CACA - - AGCTTATAATTTTGAA-GTGGTACATCAGG--AGTT - TGACTACCATTGGAT

Ca ATGGCTAACTTCAATGTATCTGTTCTTCCCCTTTTTCAAAGTTCACGT'TP - - - TTTGATT Cd GTGTTCCAATTTAGTGTATTTGTCGTTCCCCTTTC---AATTTCGTGTTTAAGTTTAATT

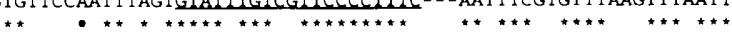

Ca GATTGATTGATTGATCTGTCGGCAGTGGTTTCAAAACCATTCGGTGAGTAATCCTATCAA Cd GATTGATTGATTGATCTGTCGGCAATGGTTTCAAA-CCATTCGGTGATAATATCATTGA

Ca TCAATGTTACGACAAAAGGCTCAATATTCAAAATTGCAATGTTTTATGTTTTCCTACGTG $\mathrm{Cd}$ TCAA TCAA--TTAAAAAACAAGGTTTAATACTTCAA-TGACAATGTTTAATGTTTTTCAACAAG

Ca TACTTGTGCAAGGCAATTGATTCAACATTGCTTTTGGTGTTTGACGAGTTTCTAGTTTGG Cd CGTTTGTGCAAATCAATTGATTCATGATTGCCTTTGATGTT - GACGAGTTTCCAATTTCG

Ca ACTTGTGTTGTTATCTGGACTATA-CAGATTTCCCGGCTCACTATGAATTTTTTTTTTTCG Cd AGTTCTG--GTTATCTGACCTATAACAGATTTCC-GGTTCATTGTAAAT-----TTTTCG

Ca ACGCTCAGTGCACACAACTATAAACAACACAAACACAAACACAGCAAGAAAAAAAAAAAC

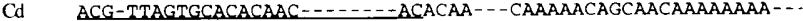

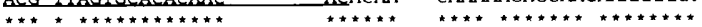

Ca GAACATTGAATTGAAACCAAGCCAACTGAAA-AATTCC -...-. TTATTTAAATGACTGT

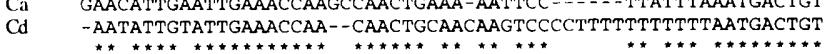

Ca CATACtaAcCCATTTTT-ATAO

Cd CGTACTMACCCATTTTTTATAC

Fig. 2. Comparison of nucleotide sequences of the $C$. albicans and $C$. dubliniensis $A C T 1$-associated introns. The $C$. albicans sequence was from strain ATCC 10123 (GenBank accession no. $X 16377$; Losberger \& Ernst, 1989) and the $C$. dubliniensis sequence from strain CD36 (EMBL accession no. AJ236897; this study). The 5 ' intron consensus sequence (GTATG), the $3^{\prime}$ intron consensus sequence (YAG) and the branchpoint sequence (TACTAAC) are in bold type. Asterisks indicate identical nucleotides and dashes indicate deletions. Sequences of the C. dubliniensis-specific primers DUBF/DUBR (Table 1) are underlined.

(Perkin Elmer Cetus) with 30 cycles of 1 min at $94{ }^{\circ} \mathrm{C}, 1 \mathrm{~min}$ at $52^{\circ} \mathrm{C}$ and $1 \mathrm{~min}$ at $72{ }^{\circ} \mathrm{C}$, followed by $72{ }^{\circ} \mathrm{C}$ for $10 \mathrm{~min}$. The amplimers were purified using the Wizard PCR Preps DNA Purification system (Promega) and cloned into pBluescript using standard procedures (Sambrook et al., 1989).

DNA sequencing. This was performed by the dideoxy chainterminating method of Sanger et al. (1977) using an automated Applied Biosystems 370A DNA sequencer and dye-labelled terminators (ABI Prism Dye Terminator Cycle Sequencing Ready Reaction kit, Applied Biosystems). Searches of the GenBank database for nucleotide sequence similarities were performed using the BLAST family of computer programs (Altschul et al., 1990). Nucleotide sequence alignments were carried out using the CLUSTAL $\mathrm{W}$ sequence analysis program (Thompson et al., 1994).

PCR identification of $C$. dubliniensis. PCR identification of $C$. dubliniensis using the C. dubliniensis-specific primer pair DUBF/DUBR (Table 1) was carried out in a $50 \mu$ l final volume containing $10 \mathrm{pmol}$ each of the forward and reverse primers, $2.5 \mathrm{mM} \mathrm{MgCl}_{2}, 10 \mathrm{mM}$ Tris $/ \mathrm{HCl}$ ( $\mathrm{pH} \mathrm{9.0} \mathrm{at} 25^{\circ} \mathrm{C}$ ), $10 \mathrm{mM}$ $\mathrm{KCl}, 0 \cdot 1 \%(\mathrm{v} / \mathrm{v})$ Triton X-100, $2 \cdot 5 \mathrm{U}$ Taq DNA polymerase 
(Promega) and $25 \mu \mathrm{l}$ template-DNA-containing cell supernatant (prepared as described above). Each reaction mixture also contained $10 \mathrm{pmol}$ each of the universal fungal primers RNAF/RNAR (Fell, 1993), which amplify approximately $610 \mathrm{bp}$ from all fungal large-subunit rRNA genes and were used as an internal positive control. Cycling conditions consisted of $6 \mathrm{~min}$ at $95^{\circ} \mathrm{C}$ followed by 30 cycles of $30 \mathrm{~s}$ at $94^{\circ} \mathrm{C}, 30 \mathrm{~s}$ at $58^{\circ} \mathrm{C}, 30 \mathrm{~s}$ at $72{ }^{\circ} \mathrm{C}$, followed by $72{ }^{\circ} \mathrm{C}$ for 10 min. Amplification products were separated by electrophoresis through $2.0 \%(\mathrm{w} / \mathrm{v})$ agarose gels containing $0.5 \mu \mathrm{g}$ ethidium bromide $\mathrm{ml}^{-1}$ and were visualized on a UV transilluminator.

\section{RESULTS}

\section{Isolation and sequence analysis of the $C$. dubliniensis ACT1 gene}

A C. dubliniensis CD36 genomic library, constructed in bacteriophage $\lambda$ EMBL3, was screened by plaque hybridization with a radioactively labelled probe consisting of the entire C. albicans ACT1 (CaACT1) gene cloned into
pBR322 (p1002, a gift from B. Magee, University of Minnesota). Approximately 10000 recombinant plaques were screened, from which 10 p1002-reactive plaques were detected. The plaque with the strongest signal was selected and subjected to single-plaque purification. DNA was extracted from the recombinant phage, termed $\lambda$ CDACT1, and restriction endonuclease digestion analysis and Southern hybridization analysis showed that $\lambda$ CDACT1 contained a DNA insert of approximately $15 \mathrm{~kb}$. Attempts to subclone smaller hybridizing fragments from the cloned insert DNA of $\lambda$ CDACT1 into the vector phagemid pBluescript, to facilitate the sequencing of the C. dubliniensis ACT1 (CdACT1) gene, failed. A number of p1002-reactive recombinant plasmids were obtained. However, upon further investigation these were shown to contain both $A C T 1$ and EMBL3 vector homologous sequences, and no recombinant plasmids harbouring only ACT1-homologous DNA were obtained. It was concluded that the $A C T 1$-homologous insert DNA from $\lambda$ CDACT1 was

Table 2. Yeast species and strains used in the phylogenetic analysis of $C$. dubliniensis

\begin{tabular}{|c|c|c|}
\hline Yeast strain & $A C T 1$ intron sequence $\dagger$ & Reference \\
\hline \multicolumn{3}{|l|}{ C. albicans } \\
\hline $132 \mathrm{~A}$ & This study & Gallagher et al. (1992) \\
\hline $179 \mathrm{~B}$ & This study & Gallagher et al. (1992) \\
\hline ATCC 10123 & $\mathrm{X} 16377$ & Losberger \& Ernst (1989) \\
\hline \multicolumn{3}{|l|}{ C. dubliniensis } \\
\hline CD36 (Ireland) & AJ236897; this study & Sullivan et al. (1995) \\
\hline CD91 (Ireland) & This study & This study \\
\hline CD70 (UK) & This study & Sullivan et al. (1997) \\
\hline NCPF 3108 (UK) & This study & Sullivan et al. (1995) \\
\hline CD93 (Finland) & This study & This study \\
\hline 94191 (Spain) & This study & Pinjon et al. (1998) \\
\hline P2 (Switzerland) & This study & Boerlin et al. (1995) \\
\hline CD71 (Argentina) & This study & Sullivan et al. (1997) \\
\hline CM2 (Australia) & This study & Sullivan et al. (1995) \\
\hline CD92 (Canada) & This study & This study \\
\hline \multicolumn{3}{|l|}{ C. glabrata } \\
\hline ATCC 90876 & AF069746 & Unpublished data submitted to GenBank \\
\hline \multicolumn{3}{|l|}{ C. stellatoidea } \\
\hline ATCC 11006 & AJ237919; this study & Kwon-Chung et al. (1989) \\
\hline 303530 & This study & bioMérieux $\ddagger$ \\
\hline 303531 & This study & bioMérieux $\ddagger$ \\
\hline \multicolumn{3}{|l|}{ C. tropicalis } \\
\hline NCPF 3111 & AJ237918; this study & NCPF catalogue \\
\hline \multicolumn{3}{|l|}{ K. lactis } \\
\hline $\mathrm{J} 7$ & M25826 & Deshler et al. (1989) \\
\hline \multicolumn{3}{|l|}{ S. cerevisiae } \\
\hline A364A & L00026 & Gallwitz \& Sures (1980) \\
\hline
\end{tabular}

* Abbreviations: ATCC, American Type Culture Collection, (Manassas, VA, USA); NCPF, National Collection of Pathogenic Fungi, Bristol, UK. The country of origin of the C. dubliniensis isolates is shown in parentheses.

† Accession numbers are for the EMBL/GenBank nucleotide sequence databases.

$\ddagger$ From the culture collection of bioMérieux, St Louis, MO, USA. 
unstable when cloned in pBluescript. Therefore it was decided to amplify CdACT1 from the recombinant phage by PCR using a mixture containing Taq polymerase and the proof-reading polymerase Pwo (Expand high-fidelity PCR system, Boehringer) and three primer sets homologous to regions of the CaACT1 gene and flanking sequences, including $5^{\prime} \mathrm{F} / 5^{\prime} \mathrm{R}, \mathrm{ACTF} / \mathrm{ACTR}$ and $3^{\prime} \mathrm{F} / 3^{\prime} \mathrm{R}$ (Table 1, Fig. 1). The three amplimers, containing overlapping sequences, obtained following PCR with these primers were cloned into pBluescript to yield recombinant plasmids $\mathrm{p} 5, \mathrm{pACT}$ and $\mathrm{p} 3$, respectively (Fig. 1). The insert DNA cloned in $\mathrm{p} 5$ and $\mathrm{p} 3$ was sequenced fully in both directions using universal primers, while the insert DNA cloned in pACT was sequenced fully by primer walking. These three overlapping sequences yielded a contiguous sequence of $1827 \mathrm{bp}$ revealing an ORF of $1131 \mathrm{bp}$ interrupted by a single $632 \mathrm{bp}$ intron at the $5^{\prime}$ end (Fig. 1). The overall nucleotide sequence identity between this ORF (CdACT1) and the CaACT1 gene was $90.6 \%$. This divergence is mainly due to differences between the intron sequences, which are $83.4 \%$ identical (Fig. 2), while the spliced coding sequences, which are identical in length in both species, are $97.9 \%$ identical. The differences between the exon sequences correspond to a total of 24 base changes. However, only one of these base substitutions $[A \rightarrow G$, at position 660 , numbering the sequences in the $5^{\prime}-3^{\prime}$ direction from the first base $(+1)$ of the translation start codon of CdACT1], results in a change in the predicted amino acid sequence, a conservative substitution from isoleucine to valine. At $632 \mathrm{bp}$ the CdACT1 intron is $25 \mathrm{bp}$ shorter than the corresponding sequence in CaACT1; however, it is situated in exactly the same position at the $5^{\prime}$ end of the gene and is recognizable by the presence of yeast intron consensus sequences (Fig. 2). These are the $5^{\prime}$ consensus sequence GTATG, the $3^{\prime}$ consensus sequence YAG, and the branchpoint sequence TACTAAC located near the $3^{\prime}$ end of the intron which is essential for efficient splicing (Mount, 1982; Langford et al., 1984; Leer et al., 1984; Molenaar et al., 1984; Teem et al., 1984).

In order to determine the level of intraspecies intron sequence conservation in epidemiologically unrelated isolates from geographically divergent parts of the world the introns of nine additional C. dubliniensis isolates, two additional C. albicans isolates (132A and 179B) and three C. stellatoidea isolates (Table 2) were amplified using the primer set INTF/INTR, which were complementary to $\mathrm{CaACT1}$ sequences flanking the intron (Table 1). The intron sequences of the 10 C. dubliniensis isolates tested, including CD36 (Table 2), were found to be very highly conserved, with only one or two base changes found within each isolate. Similar intraspecies sequence conservation was observed with the C.albicans and C. stellatoidea strains studied.

\section{Phylogenetic analysis based on ACT1 sequences}

The ACT1 gene has been used extensively to infer interspecies relationships across broad evolutionary distances (Zakut et al., 1982; Mertins \& Gallwitz, 1987;
Table 3. Genetic distance matrix based on comparison of $A C T 1$ gene coding sequences

Values correspond to percentages of difference corrected for multiple base changes by the method of Jukes \& Cantor (1969). The ACT1 gene coding sequences used were as follows: C. albicans ATCC 10123 (X16377; Losberger \& Ernst, 1989); C. dubliniensis CD36 (A J236897; this study); S. cerevisiae A364A (L00026; Gallwitz \& Sures, 1980); C. glabrata NCPF 90876 (AF069746; O. Kurzai and others, unpublished data) and K. lactis J7 (M25826; Deshler et al., 1989). Neither the C. tropicalis nor the C. stellatoidea ACT1 coding sequences are currently available in the databases, so they could not be compared with the sequences of the other yeast species used to construct the matrix.

\begin{tabular}{|lccccc|}
\hline & C. al. & C. du. & S.ce. & C. $g l$. & K. la. \\
\hline C. albicans & - & & & & \\
C. dubliniensis & $2 \cdot 1$ & - & & & \\
S. cerevisiae & $12 \cdot 9$ & $12 \cdot 6$ & - & & \\
C. glabrata & $13 \cdot 2$ & $12 \cdot 3$ & $9 \cdot 8$ & - & \\
K. lactis & $13 \cdot 2$ & $12 \cdot 6$ & $10 \cdot 1$ & $7 \cdot 8$ & - \\
\hline
\end{tabular}

Wildeman et al., 1988; Fletcher et al., 1994; Cox et al., 1995; Wery et al., 1996). This part of the study was undertaken to confirm the phylogenetic position of $C$. dubliniensis in relation to other yeast species using ACT1 sequences. This is the first time that the phylogeny of $C$. dubliniensis has been investigated using nonrRNA gene sequences. Since the ACT1 gene of many yeast species contains highly conserved (i.e. exon) and less well-conserved (i.e. intron) sequences, these regions were compared separately. Firstly, the ACT1 spliced coding sequences of C. albicans, C. dubliniensis, C. glabrata, Kluyveromyces lactis and Saccharomyces cerevisiae, obtained in this study or from the databases (Table 3), were compared using the CLUSTAL w sequence alignment software package. Secondly, the ACT1associated intron sequences from selected strains of $C$. albicans, C. dubliniensis, C. stellatoidea, C. tropicalis, C. glabrata and K. lactis (Table 4) were obtained either from GenBank or following amplification using the INTF/INTR primer set (Table 1) and also compared using CLUSTAL W. An evolutionary distance matrix for each group of sequences was generated incorporating corrections for multiple base changes according to the method of Jukes \& Cantor (1969) (Tables 3 and 4). These data indicated that the C. dubliniensis coding and intron sequences differ from the corresponding $C$. albicans sequences by $2 \cdot 1 \%$ and $16.6 \%$, respectively. Evolutionary trees constructed using the neighbourjoining method of Saitou \& Nei (1987) based on these data are shown in Fig. 3. These trees and the bootstrap values determined for each node unequivocally confirmed the unique species designation of C. dubliniensis and its phylogenetic position in relation to the other yeast species, including C. albicans. In addition, these data also confirm that C. albicans and C. stellatoidea are 
Table 4. Genetic distance matrix based on comparison of ACT1-associated intron sequences

Values correspond to percentages of difference corrected for multiple base changes by the method of Jukes \& Cantor (1969). The intron sequences used were as follows: C. albicans ATCC 10123 (X16377; Losberger \& Ernst, 1989); C. stellatoidea ATCC 11006 (AJ237919, this study); C. dubliniensis CD36 (AJ236897; this study); C. tropicalis NCPF 3111 (AJ237918, this study); C. glabrata NCPF 90876 (AF069746; unpublished data submitted to GenBank) and K. lactis J7 (M25826; Deshler et al., 1989). The sequence of the S. cerevisiae ACT1-associated intron (L00026; Gallwitz \& Sures, 1980) was not included in the construction of the matrix because it was only $308 \mathrm{bp}$ in length, significantly shorter than the intron sequences of the other yeasts studied, and so valid genetic distance determinations with this sequence and the others used to construct the matrix could not be made.

\begin{tabular}{|lcccccc|}
\hline & C. al. & C. st. & C. du. & C. tr & C. $g l$. & K. la. \\
\hline C. albicans & - & & & & & \\
C. stellatoidea & $0 \cdot 2$ & - & & & \\
C. dubliniensis & $16 \cdot 6$ & $16 \cdot 6$ & - & & \\
C. tropicalis & $43 \cdot 4$ & $43 \cdot 5$ & $47 \cdot 1$ & - & - & \\
C. glabrata & $54 \cdot 8$ & $55 \cdot 0$ & $57 \cdot 1$ & $54 \cdot 0$ & $63 \cdot 1$ & - \\
K. lactis & $58 \cdot 1$ & $58 \cdot 3$ & $54 \cdot 7$ & $61 \cdot 4$ & 6 \\
\hline
\end{tabular}

(a)

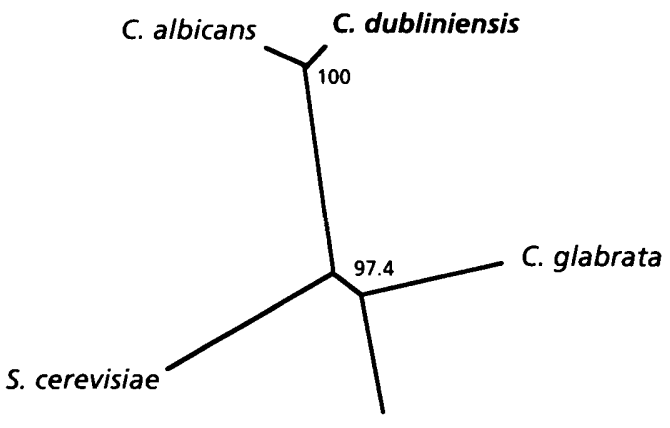

K. lactis (b)

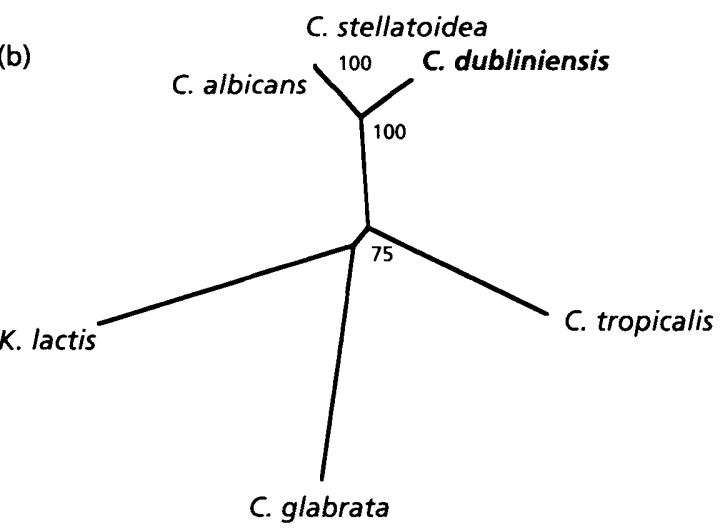

Fig. 3. Unrooted phylogenetic neighbour-joining trees generated from the alignment of the ACT1-exon (a) and -intron (b) sequences of $C$. dubliniensis and other yeast species. Numbers at the nodes were generated by bootstrap analysis (Felsenstein, 1985) and represent the percentage of times the arrangement occurred in 1000 randomly generated trees. The sequences used to construct the trees are indicated in the legends to Tables 3 and 4.

so closely related as to be considered a single species (Kamiyama et al., 1989; Sullivan et al., 1995).

\section{PCR-based identification of C. dubliniensis}

Because of the many phenotypic similarities shared by C. albicans and C. dubliniensis it is not easy to discriminate between isolates of these species in the clinical laboratory. However, examination of an alignment of the ACT1-associated intron sequences of these two species (Fig. 2) and the observation that they differ by $16.6 \%$ (Table 4 ) suggested that this significant sequence divergence could be exploited as a means to identify C. dubliniensis accurately and rapidly in combination with a rapid template DNA preparation procedure. PCR primers specific for the C. dubliniensis intron (DUBF/DUBR; Table 1, Fig. 1) were synthesized and used to amplify a DNA fragment of 288 bp from $C$. dubliniensis template DNA obtained by boiling cells from a single 48 h colony suspended in $50 \mu \mathrm{l}$ water for 10 min. PCR reactions also contained the fungal universal primers RNAF/RNAR (Fell, 1993), which amplify a product of approximately $610 \mathrm{bp}$ from the fungal largesubunit rRNA gene and serve as an internal positive control. While all fungal species should produce a product of approximately $610 \mathrm{bp}$ with the RNAF/ RNAR primers, only C. dubliniensis isolates should yield the $288 \mathrm{bp}$ amplimer with the DUBF/DUBR primer set. The C. dubliniensis-specific primer pair DUBF/ DUBR was tested in a blind trial using template DNA 
Table 5. Yeast species used in PCR identification experiments with the $C$. dubliniensis-specific primers DUBF/DUBR

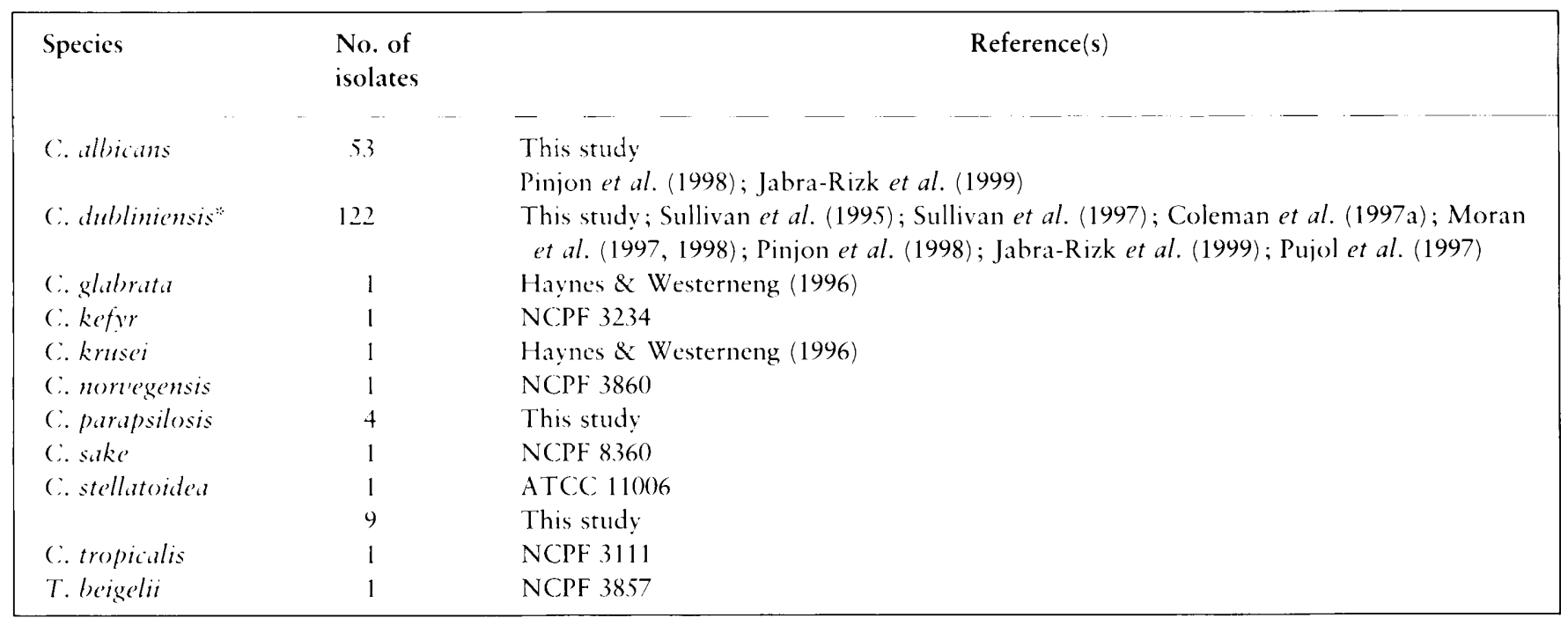

"One hundred and fourteen of the C. dubliniensis isolates were recovered from oral specimens, five were recovered from faecal specimens and one each was recovered from a vaginal, sputum and a post-mortem lung specimen. The isolates were recovered from different countries as follows: Argentina, 1 isolate; Australia, 2; Belgium, 5 ; Canada, 6; France, 4; Germany, 4; Greece, 1 ; Ireland, 48; Scandinavia, 4; Spain, 5; Switzerland, 4; UK, 17; USA, 21.

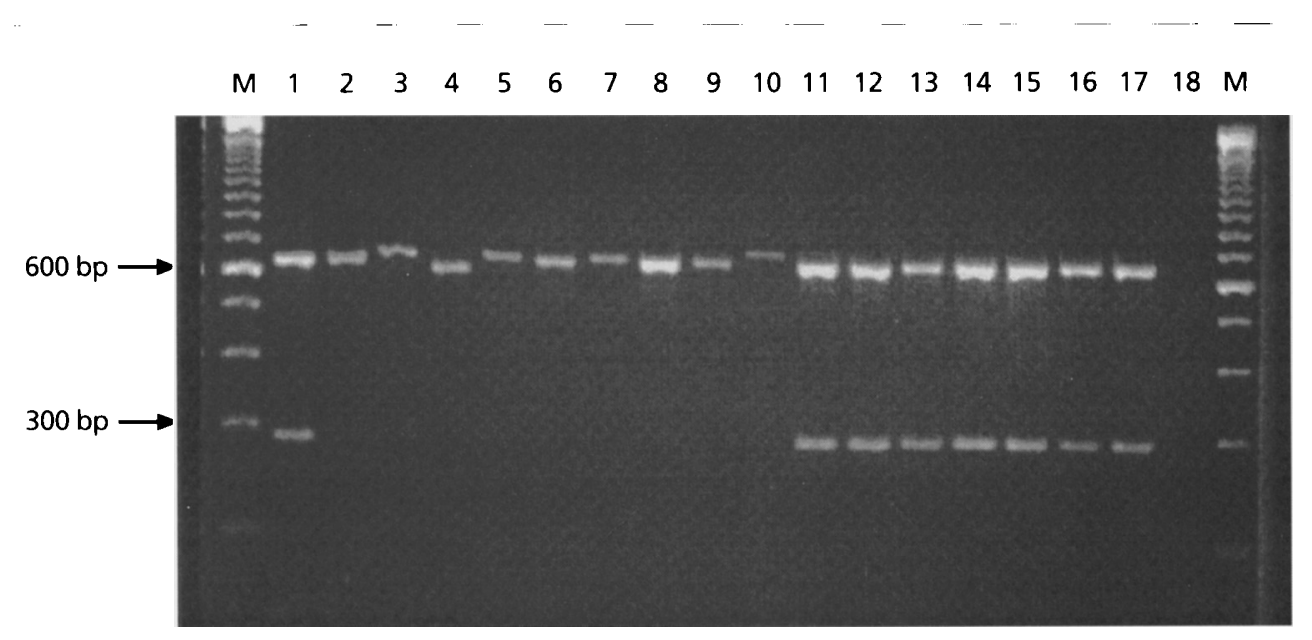

Fig. 4. Agarose gel electrophoresis of $P C R$-amplified DNA products generated using the $C$. dubliniensis-specific primers DUBF/DUBR (Table 1, Fig. 2) and the universal fungal primers RNAF/RNAR (Fell, 1993) with template DNA from yeast strains. The profiles shown correspond to: the $C$. dubliniensis type strain CD36 (lane 1); C. albicans (lane 2); C. glabrata (lane 3); C. kefyr (lane 4); C. krusei (lane 5); C. norvegensis (lane 6); C. sake (lane 7); C. stellatoidea (lane 8); C. tropicalis (lane 9): Trichosporon beigelii (lane 10); C. dubliniensis American isolate (lane 11); C. dubliniensis Argentinian isolate (lane 12); C. dubliniensis Australian isolate (lane 13); C. dubliniensis Canadian isolate (lane 14); C. dubliniensis French isolate (lane 15); C. dubliniensis German isolate (lane 16); C. dubliniensis UK isolate (lane 17). A negative control in which no template DNA was used in the PCR reaction was also included (lane 18). The $288 \mathrm{bp} \mathrm{C}$. dubliniensis-specific amplimer generated by the DUBF/DUBR primers is present in lane 1 and lanes 11-17. Lanes marked $M$ contain 100 bp size reference markers.

from the yeast isolates listed in Table 5 as follows: $C$. albicans $(n=53)$, C. dubliniensis $(n=122)$, C. glabrata $(n=1), C$. kefyr $(n=1), C$. krusei $(n=1)$, C. norvegensis $(n=1)$, C. parapsilosis $(n=4)$, C. sake $(n=1), C$. stellatoidea $(n=10), \quad$ C. tropicalis $(n=1)$ and Trichosporon beigelii $(n=1)$. All 196 yeast isolates yielded an amplimer of approximately $610 \mathrm{bp}$, but only the $C$. dubliniensis isolates yielded the 288 bp amplimer. Fig. 4 shows examples of the PCR amplimers obtained with representative strains belonging to a variety of different yeast species, including epidemiologically unrelated C. dubliniensis isolates from disparate geographical locations. Use of this PCR test in conjunction with the rapid template DNA preparation procedure used here means that a $C$. dubliniensis isolate can be identified unequivocally in less than $4 \mathrm{~h}$. 


\section{DISCUSSION}

Because C. dubliniensis was only described recently it is important to further investigate and confirm its taxonomic and phylogenetic relationship to other medically important yeast species, especially the closely related $C$. albicans. The close relationship between $C$. dubliniensis and C. albicans has resulted in difficulties in developing rapid and reliable identification techniques capable of definitively discriminating between the two species. As a result, in-depth epidemiological studies on the prevalence of this organism have been hampered. There is an urgent requirement for a test which can rapidly and definitively identify $C$. dubliniensis directly following primary culture from clinical specimens. Such a test must be simple to use, inexpensive, easily accessible to clinical diagnostic laboratories and suitable for application to large numbers of samples.

The objectives of this study were to investigate the phylogenetic relationship of C. dubliniensis to other yeast species using non-rRNA gene sequences, and to develop a rapid identification technique for this organism. To achieve these objectives the ACT1 gene was chosen for investigation, firstly because it is ideal for inferring phylogenetic relationships due to its high degree of sequence conservation in all eukaryotes, and secondly because it is unusual among yeast genes in that it is interrupted by an intron in most yeasts studied. Cloning and gene sequence analysis revealed that the overall similarity between the C. dubliniensis and C. albicans ACT1 genes is $90.6 \%$. Comparison of the spliced coding sequences of the two species revealed that the exon sequences are $97.9 \%$ identical. This level of homology is comparable to the percentage sequence identity between the two species reported previously for the V3 variable region of the large-subunit rRNA gene (97.52-97.75\% ; Sullivan et al., 1995, 1997) and the small-subunit rRNA gene $(98.6 \%$; Gilfillan et al., 1998). The predicted C. dubliniensis ACT1 protein sequence was identical to that of $C$. albicans, apart from a single conservative substitution. A phylogenetic tree generated from nucleotide comparisons of $A C T 1$ coding sequences from $C$. dubliniensis and a variety of yeast species showed that $C$. dubliniensis was grouped separately from C. albicans and the other yeast species in $100 \%$ of trees generated (Fig. 3a). These studies represent the first phylogenetic investigation of C. dubliniensis based on non-rRNA gene sequences, and they unequivocally confirm its unique position as a separate taxon within the genus Candida as determined previously by comparative rRNA gene sequence analysis (Sullivan et al., 1995, 1997; Gilfillan et al., 1998). In contrast to the highly conserved nature of the C. dubliniensis and C. albicans ACT1 exon sequences there was considerable divergence $(16.6 \%)$ between the ACT1-associated introns of the two species. When these and the ACT1associated intron sequences from a number of other yeast species were used to generate a second phylogenetic tree (Fig. 3b) the unique position of C. $d u b$ liniensis as a separate taxon within the genus Candida was affirmed. These results also confirmed that $C$. dubliniensis is most closely related to C. albicans. In addition, the C. albicans and C. stellatoidea ACT1associated introns were found to differ by one basepair substitution, corresponding to a $0.2 \%$ sequence divergence. These findings provided further evidence that C. albicans and C. stellatoidea should be considered as the same species. This situation is analogous to that found between $S$. cerevisiae and S. carlsbergensis, where the ACT1-associated introns differ by one basepair deletion and one basepair substitution, and it is accepted that these two organisms are in fact the same species (Nellen et al., 1981). The C. tropicalis intron sequence differs from that of C. albicans by $43.4 \%$, confirming that it is more distantly related to $C$. albicans than $C$. dubliniensis (Table 4, Fig. 3b). All of these findings indicate that the $A C T 1$-associated intron sequences are not subject to the same level of evolutionary constraint as the $A C T 1$ coding sequences.

The ACT1 genes of fungal species, in general, are noteworthy because of the presence of introns (Gallwitz \& Sures, 1980; Fidel et al., 1988; Wildeman et al., 1988; Deshler et al., 1989; Losberger \& Ernst, 1989; Fletcher et al., 1994; Cox et al., 1995; Matheucci et al., 1995; Wery et al., 1996). At present, most known introns can be assigned unambiguously to one of four classes, depending on the intron structure and location (Krainer \& Maniatis, 1988). ACT1-associated introns belong to class IV, which are nuclear pre-mRNA introns. The $C$. dubliniensis ACT1-associated intron is located at the 5' end of the gene, where it interrupts the fourth codon. The ACT1 genes of C. albicans, C. glabrata, S. cerevisiae and $K$. lactis all contain introns located at this codon (Losberger \& Ernst, 1989; O. Kurzai and others, unpublished data submitted directly to GenBank accession no. AF069746); Gallwitz \& Sures, 1980; Deshler et al., 1989). This position is conserved amongst fungi, as all fungal actin genes that contain an intron do so at the third, fourth or fifth codon. Three conserved sequence elements have also been identified in the nuclear pre-mRNA introns of yeasts, at the $5^{\prime}$ and $3^{\prime}$ splice sites and at a site within the intron near the $3^{\prime}$ splice site, known as the branchpoint sequence. All three conserved elements have been shown to be important for the accurate and efficient splicing of introns in $S$. cerevisiae (Langford et al., 1984; Leer et al., 1984; Molenaar et al., 1984; Teem et al., 1984; Mount, 1982). The C. dubliniensis, C. albicans, C. stellatoidea and C. tropicalis ACT1-associated introns possess all three conserved elements, namely GTATG $\left(5^{\prime}\right.$ consensus $)$, TAG ( $3^{\prime}$ consensus) and TACTAAC (branchpoint), (this study, Fig. 2; Losberger \& Ernst, 1989). These sequences are also present in C. glabrata and $K$. lactis although the $3^{\prime}$ consensus sequence is CAG (Deshler $e t$ al., 1989; see GenBank accession no. AF069746 for the C. glabrata ACT1-intron sequence).

One striking feature of the C. dubliniensis introns was that they showed little intraspecies variation, even among isolates from geographically divergent locations. The small changes which were recorded consisted of single base changes, some of which were shared by more 
than one strain, and deletions which occurred at the end of poly $(\mathrm{T})$ and $\operatorname{poly}(\mathrm{A})$ stretches. Introns containing these deletions were sequenced on separate occasions using different preparations of template DNA to rule out the possibility of sequencing or amplification artefacts. We concluded that these deletions are genuine and are probably the result of slipped-strand mispairing during replication. Similar intraspecies sequence conservation was observed with the ACT1-associated introns from $C$. albicans and C. stellatoidea. Boucher et al. (1996) made similar findings with their analysis of the group I selfsplicing intron present in the large-subunit rRNA gene, in which the intron is present in a similar location in $C$. albicans, C. stellatoidea and C. dubliniensis. Again there was no significant intraspecies variation in the intron sequence. Furthermore, the C. albicans self-splicing intron and that of C. stellatoidea showed a high degree of homology, differing only by three single basepair substitutions. They also found that the homology between the C. albicans and C. dubliniensis group I introns (CaLSU and CdLSU, respectively) was quite high except for two regions of divergence contained in two stem-loop regions, both of which are much longer in C. dubliniensis than in C. albicans. These two regions lie outside the catalytic core, and although they are predicted to have a more complex secondary structure than those of $C$. albicans they do not affect the selfsplicing ability of the intron. Our analysis of the $C$. dubliniensis and C. albicans ACT1-associated introns showed that although identical conserved elements are present in both species, nucleotide differences accounting for a $16.6 \%$ sequence divergence were dispersed throughout the length of the intron (Fig. 2). With group I introns, conservation of the nucleotide sequence may be important as it dictates the secondary structure of the intron and therefore its self-splicing ability. However, with group IV introns, such as the C. dubliniensis and C. albicans ACT1-associated introns, the splicing event is mediated by the spliceosome and although maintenance of the three conserved elements is important for splicing there do not appear to be any other constraints upon conservation of the nucleotide sequence. This may explain why divergence between the C. dubliniensis and C. albicans ACT1-associated introns sequences is dispersed throughout the intron.

Genotypic tests such as DNA fingerprinting analysis, karyotype analysis and RFLP analysis have been used in the differentiation of $C$. dubliniensis and C. albicans isolates. However, these techniques cannot be easily applied to the analysis of large numbers of clinical isolates. In contrast, PCR, which may be applied to the detection of genetic differences, is rapid and relatively inexpensive. The low level of intraspecies sequence variation in the C.dubliniensis $A C T 1$-associated intron, and the extent of divergence from the C. albicans ACT1associated intron sequence, suggested that this region could provide the basis for the design of oligonucleotide primers capable of readily discriminating between isolates of both species using PCR. In order to facilitate the rapid processing of large numbers of samples and to decrease the time required, template DNA was prepared by boiling a single fresh Candida colony suspended in $50 \mu \mathrm{l}$ water. With template DNA preparation taking approximately $15 \mathrm{~min}$, PCR amplification $2.5 \mathrm{~h}$ and electrophoresis of amplimers $1 \mathrm{~h}, \mathrm{C}$. dubliniensis colonies can be identified in as little as $4 \mathrm{~h}$. Thus $C$. dubliniensis colonies can be identified directly from primary isolation plates without the requirement for additional subculture. This technique is particularly effective when used for the identification of presumptive C. dubliniensis isolates cultured from clinical specimens on CHROMagar Candida medium. The C. dubliniensisspecific primers were tested with a collection of 122 isolates of C. dubliniensis in a blind trial with isolates of 10 other yeast species, including 53 isolates of C. albicans and 10 isolates of C. stellatoidea (Table 5). The primers correctly identified only the C. dubliniensis isolates, with $100 \%$ accuracy. Recently, Mannarelli \& Kurtzman (1998) also developed a PCR-based identification test for discriminating between C. albicans and C. dubliniensis isolates; however, these primers were only tested with seven C. dubliniensis isolates. Another study by Elie $e t$ al. (1998) reported the development of a C. dubliniensis probe, specific for the internal transcribed spacer region (ITS2) of the ribosomal gene cluster. However, this probe has been tested with a very small sample number $(n=5)$ and the method itself involves a PCR-enzymelinked immunoassay, which is relatively time consuming. In contrast, our method is a simple and rapid technique capable of identifying suspect colonies directly from a primary isolation plate. In addition it has been evaluated against a large number of isolates from diverse geographical locations. Our findings clearly demonstrate that PCR identification based upon the ACT1associated intron sequence is a definitive and rapid technique for the identification of C. dubliniensis.

\section{ACKNOWLEDGEMENTS}

This work was supported by a grant from the Wellcome Trust (no. 047204). We thank Dr B. Magee, University of Minnesota, for providing the C. albicans ACT1-encoding plasmid p1002. We thank our colleagues who sent us isolates of Candida dubliniensis, including Aristea Velegraki, National University of Athens, Greece; Markus Ruhnke, Virchow Klinikum der Humboldt Universität, Berlin, Germany; Luc Giasson, Laval University, Québec, Canada; Jose Ponton, Universidad del País Vasco, Bilbao, Spain; Elizabeth Johnson, Public Health Laboratory Service, Mycology Reference Laboratory, Bristol, UK; Frank Odds, Janssen Research Foundation, Beerse, Belgium; Lakshman Samaranayake, University of Hong Kong; Mary Ann Jabra-Rizk, Johns Hopkins University, Baltimore, USA; Jean-Marie Bastide, Université de Montpellier, France; Fiona Mulcahy, St. James's Hospital, Dublin, Ireland; and Christine McCreary, Dublin Dental Hospital, Dublin, Ireland.

\section{REFERENCES}

Altschul, S. F., Gish, W., Miller, W., Myers, E. W. \& Lipman, D. J. (1990). Basic local alignment search tool. J Mol Biol 215, 403-410.

Bennett, D. E., McCreary, C. E. \& Coleman, D. C. (1998). Genetic characterization of a phospholipase $\mathrm{C}$ gene from Candida 
albicans: presence of homologous sequences in Candida species other than C. albicans. Microbiology 144, 55-72.

Boerlin, P., Boerlin-Petzold, F., Durussel, C., Addo, M., Pagani, J.-L., Chave, J.-P. \& Bille, J. (1995). Cluster of atypical Candida isolates in a group of human immunodeficiency virus-positive drug users. J Clin Microbiol 33, 1129-1135.

Boucher, H., Mercure, S., Montplaisir, S. \& Lemay, G. (1996). A novel group I intron in Candida dubliniensis is homologous to a Candida albicans intron. Gene 180, 189-196.

Coleman, D. C., Sullivan, D. J., Bennett, D. E., Moran, G. P., Barry, H. J. \& Shanley, D. B. (1997a). Candidiasis: the emergence of a novel species, Candida dubliniensis. AIDS 11, 557-567.

Coleman, D., Sullivan, D., Harrington, B., Henman, M., Shanley, D., Bennett, D., Moran, G., McCreary, C. \& O'Neill, L. (1997b). Molecular and phenotypic analysis of Candida dubliniensis: a recently identified species linked with oral candidosis in HIVinfected and AIDS patients. Oral Dis 3 (Supplement 1), S96-S101.

Cox, G. M., Rude, T. H., Dykstra, C. C. \& Perfect, J. R. (1995). The actin gene from Cryptococcus neoformans: structure and phylogenetic analysis. J Med Vet Mycol 33, 261-266.

Deshler, J. O., Garrett, L. P. \& Rossi, J. J. (1989). Kluyveromyces lactis maintains Saccharomyces cerevisiae intron-encoded splicing signals. Mol Cell Biol 9, 2208-2213.

Elie, C. M., Lott, T. J., Reiss, E. \& Morrison, C. J. (1998). Rapid identification of Candida species with species-specific DNA probes. J Clin Microbiol 36, 3260-3265.

Fell, J. (1993). Rapid identification of yeast species using three primers in a polymerase chain reaction. Mol Mar Biol Biotechnol 3, 174-180.

Felsenstein, J. (1985). Confidence limits on phylogenies: an approach using the bootstrap. Evolution 39, 783-791.

Fidel, S., Doonan, J. H. \& Morris, N. R. (1988). Aspergillus nidulans contains a single actin gene which has unique intron locations and encodes a gamma actin. Gene 70, 283-293.

Fletcher, L. D., McDowell, J. M., Tidwell, R. R., Meagher, R. B. \& Dykstra, C. C. (1994). Structure, expression and phylogenetic analysis of the gene encoding actin 1 in Pneumocystis carinii. Genetics 137, 743-750.

Frischauf, A. M., Lehrach, H., Poustka, A. \& Murray, N. (1983). Lambda replacement vectors carrying polylinker sequences. $J$ Mol Biol 170, 827-842.

Gallagher, P. J., Bennett, D. E., Henman, M. C., Russell, R. J., Flint, S. R., Shanley, D. B. \& Coleman, D. C. (1992). Reduced azole susceptibility of Candida albicans from HIV-positive patients and a derivative exhibiting colony morphology variation. J Gen Microbiol 138, 1901-1911.

Gallwitz, D. \& Sures, I. (1980). Structure of a split yeast gene: complete nucleotide sequence of the actin gene in Saccharomyces cerevisiae. Proc Natl Acad Sci USA 77, 2546-2550.

Gilfillan, G. D., Sullivan, D. J., Haynes, K., Parkinson, T., Coleman, D. C. \& Gow, N. A. R. (1998). Candida dubliniensis: phylogeny and putative virulence factors. Microbiology 144, 829-838.

Haynes, K. A. \& Westerneng, T. J. (1996). Rapid identification of Candida albicans, C. glabrata, C. parapsilosis and C. krusei by species-specific PCR of large subunit ribosomal DNA. J Med Microbiol 44, 390-396.

Hennessy, E. S., Drummond, D. R. \& Sparrow, J. C. (1993). Molecular genetics of actin function. Biochem J 282, 657-671.

Hightower, R. C. \& Meagher, R. B. (1986). The molecular evolution of actin. Genetics 114, 315-332.

Jabra-Rizk, M. A., Baqui, A. A., Kelley, J. I., Falkler, W. A. J., Merz,
W. G. \& Meiller, T. F. (1999). Identification of Candida dubliniensis in a prospective study of patients in the United States. J Clin Microbiol 37, 321-326.

Jukes, T. H. \& Cantor, C. R. (1969). Evolution of protein molecules. In Mammalian Protein Metabolism, vol. 3, pp. 21-132. Edited by H. N. Munro. New York: Academic Press.

Kamiyama, A., Niimi, M., Tokunaga, M. \& Nakayama, H. (1989). DNA homology between Candida albicans strains: evidence to justify the synonymous status of Candida stellatoidea. Mycopathologia 107, 3-7.

Kirkpatrick, W. R., Revankar, S. G., McAtee, R. K. \& 7 other authors (1998). Detection of Candida dubliniensis in oropharyngeal samples from human immunodeficiency virus-infected patients in North America by primary CHROMagar Candida screening and susceptibility testing of isolates. J Clin Microbiol 36, 3007-3012. .

Korn, E. D. (1978). Biochemistry of actomyosin-dependent cell motility (a review). Proc Natl Acad Sci USA 75, 588-599.

Krainer, A. R. \& Maniatis, T. (1988). RNA splicing. In Transcription and Splicing, pp. 131-206. Edited by B. D. Hames \& D. M. Glover. Washington DC: IRL Press.

Kwon-Chung, K. J., Riggsby, W. S., Uphoff, R. A., Hicks, J. B., Whelan, W. L., Reiss, E., Magee, B. B. \& Wickes, B. L. (1989). Genetic differences between type I and type II Candida stellatoidea. Infect Immun 57, 527-532.

Langford, C. J., Klinz, F., Donath, C., Gallwitz, D. (1984). Point mutations identify the conserved intron-contained TACTAAC box as an essential splicing signal sequence in yeast. Cell 36, 645-653.

Leer, R. J., van Raamsdonk-Duin, M. M. C., Hagendoorn, M. J. M., Mager, W. H. \& Planta, R. J. (1984). Structural comparison of yeast ribosomal protein genes. Nucleic Acids Res 12, 6685-6700. Losberger, C. \& Ernst, J.F. (1989). Sequence of the Candida albicans gene encoding actin. Nucleic Acids Res 17, 9488.

Mannarelli, 8. M. \& Kurtzman, C. P. (1998). Rapid identification of Candida albicans and other human pathogenic yeasts by using short oligonucleotides in a PCR. J Clin Microbiol 36, 1634-1641.

Matheucci, E., Jr, Henrique-Silva, F., El-Gogary, S., Rossini, C. H. B., Leite, A., Escobar Vera, J., Carle Urioste, C., Crivellaro, 0. \& El-Dorry, H. (1995). Structure, organization and promoter expression of the actin-encoding gene in Trichoderma reesei. Gene 161, 103-106.

Meis, J. F., Ruhnke, M., De Pauw, B. E., Odds, F. C., Siegert, W. \& Verweij, P. E. (1999). Candida dubliniensis candidemia in patients with chemotherapy-induced neutropenia and bone marrow transplantation. Emerging Infect Dis 5, 150-153.

Mertins, P. \& Gallwitz, D. (1987). A single intronless actin gene in the fission yeast Schizosaccharomyces pombe: nucleotide sequence and transcripts formed in homologous and heterologous yeast. Nucleic Acids Res 15, 7369-7379.

Molenaar, C. M. T., Woudt, L. P., Jansen, A. E. M., Mager, W. H., Planta, R. J., Donovan, D. M. \& Pearson, N. J. (1984). Structure and organization of two linked ribosomal protein genes in yeast. Nucleic Acids Res 12, 7245-7358.

Moran, G. P., Sullivan, D. J., Henman, M. C., McCreary, C. E., Harrington, B. J., Shanley, D. B. \& Coleman, D. C. (1997). Antifungal drug susceptibilities of oral Candida dubliniensis isolates from human immunodeficiency virus (HIV)-infected and nonHIV-infected subjects and generation of stable fluconazoleresistant derivatives in vitro. Antimicrob Agents Chemother 41, $617-623$.

Moran, G. P., Sanglard, D., Donnelly, S. M., Shanley, D. B., 
Sullivan, D. J. \& Coleman, D. C. (1998). Identification and expression of multidrug transporters responsible for fluconazole resistance in Candida dubliniensis. Antimicrob Agents Chemother 42, 1819-1830.

Mount, S. M. (1982). A catalogue of splice junction sequences. Nucleic Acids Res 10, 459-472.

Nellen, W., Donath, C., Moos, M. \& Gallwitz, D. (1981). The nucleotide sequences of the actin genes from Saccharomyces carlsbergensis and Saccharomyces cerevisiae are identical except for the introns. J Mol Appl Genet 1, 239-244.

Odds, F. C., Van Nuffel, L. \& Dams, G. (1998). Prevalence of Candida dubliniensis isolates in a yeast stock collection. J Clin Microbiol 6, 2869-2873.

Pfaller, M. A., Messer, S. A., Gee, S., Joly, S., Pujol, C., Sullivan, D. J., Coleman, D. C. \& Soll, D. R. (1999). In vitro susceptibilities of Candida dubliniensis isolates tested against the new triazole and echinocandin antifungal agents. J Clin Microbiol 37, 870-872.

Pinjon, E., Sullivan, D., Salkin, I., Shanley, D. \& Coleman, D. (1998). Simple, inexpensive, reliable method for differentiation of Candida dubliniensis from Candida albicans. J Clin Microbiol 36, 2093-2095.

Pollard, T. D. (1990). Actin. Curr Opin Cell Biol 2, 33-40.

Pujol, C., Renaud, F., Mallie, M., de Meeus, T. \& Bastide, J.-M. (1997). Atypical strains of Candida albicans recovered from AIDS patients. J Med Vet Mycol 35, 115-121.

Saitou, N. \& Nei, M. (1987). The neighbour joining method for reconstructing phylogenetic trees. Mol Biol Evol 4, 406-425.

Salkin, I. F., Pruitt, W. R., Padhye, A. A., Sullivan, D., Coleman, D. \& Pincus, D. H. (1998). Distinctive carbohydrate assimilation profiles used to identify the first clinical isolates of Candida dubliniensis recovered from the United States. J Clin Microbiol 36, 1467.

Sambrook, J., Fritsch, E. F. \& Maniatis, T. (1989). Molecular Cloning: a Laboratory Manual, 2nd edn. Cold Spring Harbor, NY : Cold Spring Harbor Laboratory.

Sanger, F., Nicklen, S. \& Coulson, A. R. (1977). DNA sequencing with chain-terminating inhibitors. Proc Natl Acad Sci USA 74, $546.3-5467$.
Schoofs, A., Odds, F. C., Colebunders, R., leven, M. \& Goosens, H. (1997). Use of specialised isolation media for recognition and identification of Candida dubliniensis isolates from HIV-infected patients. Eur J Clin Microbiol Infect Dis 16, 296-300.

Sullivan, D. \& Coleman, D. (1998). Candida dubliniensis: characteristics and identification. J Clin Microbiol 36, 329-334.

Sullivan, D. J., Westerneng, T. J., Haynes, K. A., Bennett, D. E. \& Coleman, D. C. (1995). Candida dubliniensis sp. nov.: phenotypic and molecular characterisation of a novel species associated with oral candidosis in HIV-infected individuals. Microbiology 141, $1507-1521$.

Sullivan, D., Haynes, K., Bille, J., Boerlin, P., Rodero, L., Lloyd, S., Henman, M. \& Coleman, D. (1997). Widespread geographic distribution of oral Candida dubliniensis strains in human immunodeficiency virus-infected individuals. J Clin Microbiol 35, 960-964.

Thompson, J. D., Higgins, D. G. \& Gibson, T. J. (1994). CLuSTAL w : improving the sensitivity of progressive multiple sequence alignment through sequence weighting, position-specific gap penalties and weight matrix choice. Nucleic Acids Res 22, 4673-4680.

Teem, J. L., Abovich, N., Kaufer, N. F. \& 12 other authors (1984). A comparison of yeast ribosomal protein gene DNA sequences. Nucleic Acids Res 12, 8295-8312.

Welch, M. D., Holtzman, D. A. \& Drubin, D. G. (1994). The yeast actin cytoskeleton. Curr Opin Cell Biol 6, 110-119.

Wery, J., Dalderup, M. J., Ter Linde, J., Boekhout, T. \& Van Ooyen, A. J. J. (1996). Structural and phylogenetic analysis of the actin gene from the yeast Phaffia rhodozyma. Yeast 12, 641-651.

Wildeman, A. G. (1988). A putative ancestral actin gene present in a thermophilic eukaryote: novel combination of intron positions. Nucleic Acids Res 16, 2553-2564.

Zakut, R., Shanai, M., Givol, D., Neuman, S., Yaffe, D. \& Nudel, U. (1982). Nucletoide sequence of the rat skeletal muscle actin gene. Nature 298, 857-859.

Received 9 April 1999; revised 26 May 1999; accepted 27 May 1999. 